\title{
THE ECLIPSES OF THE CLOSE BINARY STAR BE UMA
}

\author{
JANET H. WOOD ${ }^{1}$, E. L. ROBINSON ${ }^{2}$, E.-H. ZHANG ${ }^{2}$ \\ 1. Physics Department, Keele University, Keele, ST5 5BG, UK \\ 2. Department of Astronomy, University of Texas, Austin, TX \\ 78712, USA
}

\section{Introduction}

BE UMa is a close binary star, not transferring mass, with an extremely hot primary star irradiating the inner face of the cool secondary star. The light curve shows a large-amplitude, sinusoidal variation with a period of $2.29 \mathrm{~d}$, and an eclipse that is centered on the minimum of the variation [1], [3]. According to [1], the eclipse is partial, not total. However, it has been argued [2] that the eclipse was really flat bottomed and thus total. This has important repercussions for the deduced model of the system. To resolve this issue we obtained simultaneous $U B V R$ photometry of BE UMa using the Stiening 4-channel, high-speed photometer on the 82-inch telescope at McDonald Observatory. The mean light curves are shown in Fig. 1. The eclipse in all colours is round bottomed and partial. The different depths are caused by the different contribution from the red secondary star in each bandpass.

\section{The system parameters}

Along with the inclination, $i$, the primary star radius, $R_{1} / a$, and the secondary star radius, $R_{2} / a$, for each bandpass the flux from the primary star, the limb darkening of the primary star, and the secondary star flux, determine the light curve. For a given inclination we fit the four light curves simultaneously. We find our best fit for $i=82.4^{\circ} \pm 0.1^{\circ}, R_{1} / a=$ $0.0781 \pm 0.0001$ and $R_{2} / a=0.138 \pm 0.001$. The number of fitted data points is 6768 and for this fit $\chi^{2}=7202$. The deduced limb darkening coefficients are very unusual: to obtain the best fit, limb brightening of the primary was required in the $U$ band with almost full limb darkening in the $B$ band.

Additional information is necessary to determine the stellar masses, $M_{1}$ and $M_{2}$, the mass ratio, $q=M_{2} / M_{1}$, and the orbital separation, $a$. Using 


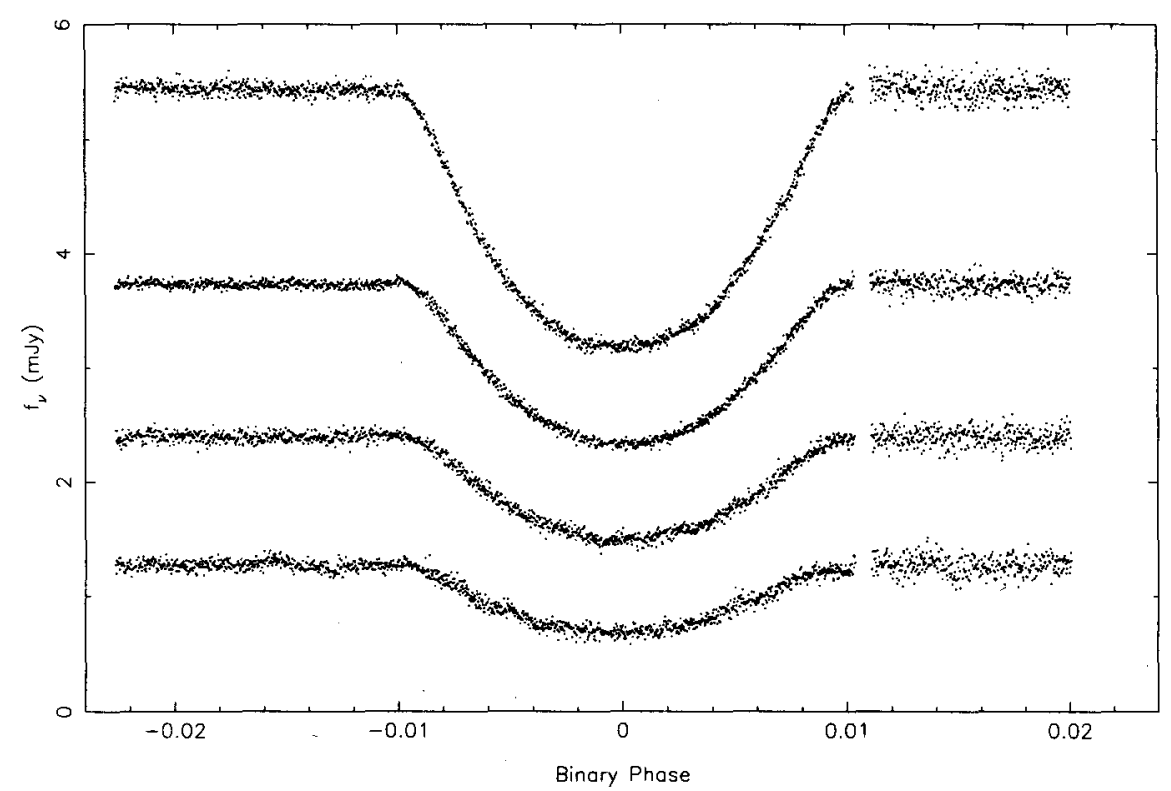

Figure 1. From bottom to top, the mean $R, V, B$ and $U$ light curves at $5 \mathrm{~s}$ time resolution. Each light curve has been offset upwards by $1 \mathrm{mJy}$ with the $R$ light curve at its true flux density.

the radial velocity of the secondary star [2], corrected for the effects of irradiation, and assuming that the secondary star lies on the main sequence, we can determine the system parameters for each inclination. For $i=82.4^{\circ}$ we find $M_{1}=1.43 \pm 0.15 \mathrm{M}_{\odot}, M_{2}=1.39 \pm 0.15 \mathrm{M}_{\odot}$ and $a=(7.2 \pm 0.3) 10^{11} \mathrm{~cm}$. We note that the primary star is a subdwarf: it is not small enough to be a white dwarf. The secondary star lies well inside its Roche lobe.

At phase zero we are seeing the unirradiated face of the secondary star and can use the measured fluxes to determine its spectral type. We find that G6-K4 is acceptable. We fit pure hydrogen, $\log g=6$, model atmospheres [4] to our primary star fluxes, The fit for $T_{\mathrm{e}}=125000 \mathrm{~K}$ corresponds to a distance of $986<D(\mathrm{pc})<1205$. If the secondary star is a K3-K4 star it has a distance consistent with the $1 \sigma$ limits deduced from the subdwarf. For a $\mathrm{K}$ dwarf secondary star, we therefore have a self-consistent model.

\section{References}

1. Ando, H., Okazaki, A., Nishimura, S., 1982, PASJ, 34, 141

2. Crampton, D., Cowley, A.P., Hutchings, J.B., 1983, Ap. J., 272, 202

3. Kurochkin, N.E., 1971, Perem. Zvezdy., 18, 85

4. Wesemael, F., Auer, L.H., van Horn, H.M., et al., 1980, Ap. J. Supp., 43, 159 